\title{
Location is everything when it comes to megakaryocyte function
}

\author{
Eric Boilard ${ }^{1,2}$ and Kellie R. Machlus ${ }^{3}$ \\ 1Department of Infectious Diseases and Immunity, Centre de Recherche du CHU de Québec, Québec, Québec, Canada. ²Université Laval and Centre de Recherche Arthrite, Québec, Québec, Canada. ${ }^{3}$ Vascular \\ Biology Program, Boston Children's Hospital, Department of Surgery, Harvard Medical School, Boston, Massachusetts, USA.
}

\begin{abstract}
Megakaryocytes (MKs) give rise to platelets, which are blood cells that are essential to prevent hemorrhage. Although the majority of MKs localize to the bone marrow, there is a distinct population of lung-residing MKs $\left(M K_{L}\right)$. In this issue of the $J C l$, Pariser et al. examined gene expression patterns of MKs collected from murine and nonhuman primate bone marrow or lung. This Commentary explores the premise that environmental factors from the lung determine the genetic and phenotypic similarity of $\mathrm{MK}_{\mathrm{L}}$ to lung dendritic cells, distinguishing $M K_{L}$ from bone marrow MKs. Indeed, while $M K_{L}$ retain the ability to make platelets, they also process and present antigens that activate $\mathrm{CD} 4^{+}$lymphocytes. These data suggest that $\mathrm{MK}_{\mathrm{L}}$ may play an important role in immune processes beyond platelet production.
\end{abstract}

\section{Megakaryocytes in the lung} Megakaryocytes (MKs) are unique cells that give rise to circulating platelets. The majority of MKs reside in the bone marrow (BM), where they differentiate from hematopoietic stem cells, mature, and release proplatelets into the vascular lumen that are quickly converted into platelets $(1,2)$. However, MKs have also been found in other organs such as the lungs, liver, and spleen $(3,4)$, with up to $8 \%$ of murine MKs residing in the lungs (5). Although lung MKs were first observed in the late 19th century, their biological function and relevance have remained obscure. In this issue of the JCI, Pariser et al. have begun to unravel the mysteries of lung-residing MKs $\left(\mathrm{MK}_{\mathrm{L}}\right)$ by uncovering their unique properties, and reveal that $\mathrm{MK}_{\mathrm{L}}$ are genetically and phenotypically similar to resident lung dendritic cells (6).

Previous findings suggest that $\mathrm{MK}_{\mathrm{L}}$ can produce platelets $(4,7)$. Although it is debated whether a substantial proportion of circulating platelets are generated from $\mathrm{MK}_{\mathrm{L}}$, the recent reemergence of this unique MK population has led to questions about if and how $\mathrm{MK}_{\mathrm{L}}$ differ from BM MKs. Pariser and colleagues isolated $\mathrm{BM}$ and lung myeloid-enriched cells from both mice and nonhuman primates and characterized the populations using single-cell RNA sequencing. The researchers found that $\mathrm{MK}_{\mathrm{L}}$ have gene expression patterns that are more similar to lung dendritic cells than autologous BM MKs. Indeed, $\mathrm{MK}_{\mathrm{L}}$ express high levels of markers such as major histocompatibility complex class II (MHC-II), CD11c, ICAM, and CCR7, with MHC-II being a marker that appears to faithfully differentiate $\mathrm{MK}_{\mathrm{L}}$ and $\mathrm{BM} \mathrm{MKs}$. In addition, the $\mathrm{MK}_{\mathrm{L}}$ population is predominantly diploid, or $2 \mathrm{~N}$, which is in stark contrast to larger BM MKs that have an average ploidy of $16 \mathrm{~N}$. Although both $\mathrm{MK}_{\mathrm{L}}$ and $\mathrm{BM} \mathrm{MKs}$ are capable of generating proplatelets in culture, the overall platelet-generating capacity of each population was not investigated. Together, these data suggest that $\mathrm{MK}_{\mathrm{L}}$ are both morphometrically

\section{Related Article: https://doi.org/10.1172/JCI137377}

Conflict of interest: The authors have declared that no conflict of interest exists.

and genotypically unique, and therefore may play an important role in processes beyond platelet production.

\section{The immune phenotype of $M K_{L}$ is plastic}

One of the more intriguing findings from Pariser et al. (6) is that the immune phenotype of $\mathrm{MK}_{\mathrm{L}}$ is plastic and driven by the tissue environment. The BM and lung have many environmental distinctions, some of the most notable being differences in the higher oxygenation and increased accessibility to pathogens in the lungs. Therefore, Pariser and colleagues aimed to determine the roles that these unique environments play in formation and/or maintenance of their resident MKs. To do this, the authors cleverly used neonatal (PO) mouse lungs to determine if the $\mathrm{MK}_{\mathrm{L}}$ phenotype changes over development. They found that PO $\mathrm{MK}_{\mathrm{L}}$ did not yet resemble the $\mathrm{MK}_{\mathrm{L}}$ in adult lungs, suggesting that this immune phenotype is not an inherent property of the MK population, but is rather gained over time as a result of the $\mathrm{MK}_{\mathrm{L}}$ environment. Likewise, the converse was also found to be true. While exposure of BM MKs to hypoxic and normoxic conditions failed to impact expression of MHC-II molecules, BM MKs had the capacity to adopt an $\mathrm{MK}_{\mathrm{L}}$-like phenotype under the influence of pathogenassociated molecular patterns and lungassociated immune molecules, such as IL-33. In vivo administration of BM MKs to the lungs further confirmed this plasticity; after 48 hours, infused BM MKs upregulated both MHC-II and CCR7. Of note, however, resident $\mathrm{MK}_{\mathrm{L}}$ secreted more immune molecules compared with BM MKs upon stimulation with lipopolysaccharide. This result suggests that the $\mathrm{MK}_{\mathrm{L}}$ programming results in functionally distinct MKs (6). What has yet to be fully addressed, however, is the source(s) of the $\mathrm{MK}_{\mathrm{L}}$ population. Are the $\mathrm{MK}_{\mathrm{L}}$ derived from hematopoietic stem cells that reside in the BM? And if not, is there a pool of resident hematopoietic 


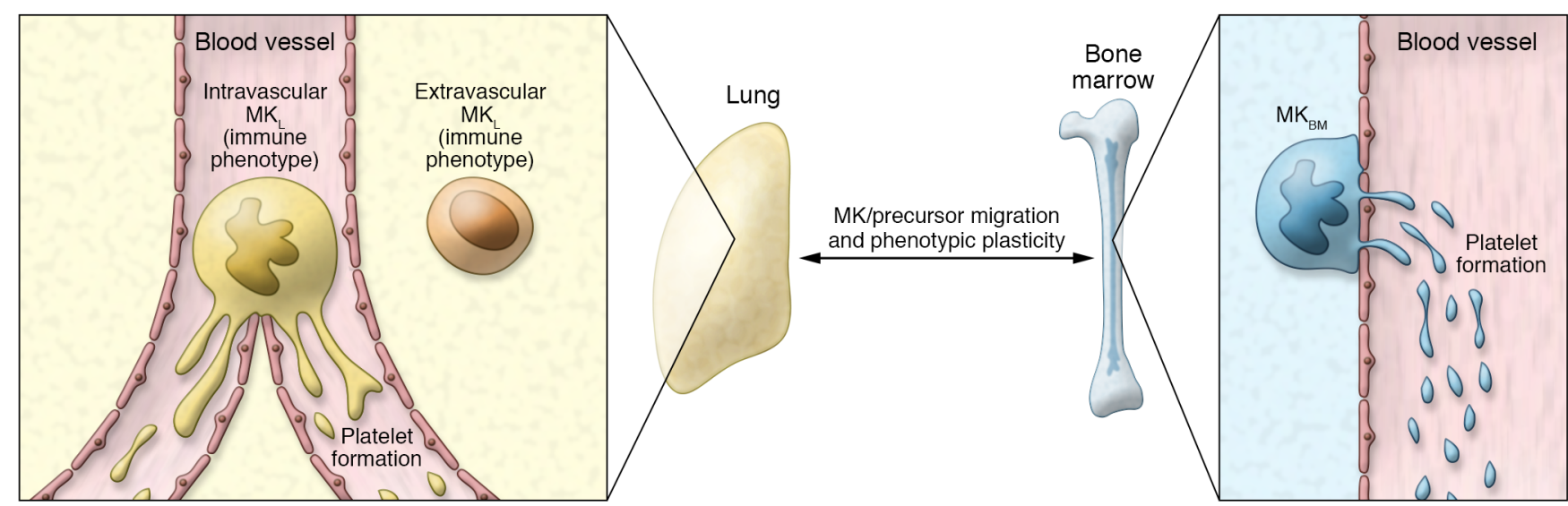

Figure 1. Model for MK plasticity. The lung contains intravascular and extravascular populations of megakaryocytes $\left(M_{L}\right)$. $M K_{L}$ that are large, polyploid (average of $16 \mathrm{~N}$ ), and intravascular are ideally positioned to release proplatelets into flowing blood. Extravascular diploid (2N) MK $K_{L}$ resemble lung dendritic cells. The local environment modulates the phenotype of $B M M K_{s}\left(M_{B M}\right)$ and $M_{K_{L}}$. Notably, $M_{B M}$ that are transferred into the lungs adopt an $M K_{L}$-like phenotype (6). MKs or MK progenitors may continuously migrate between the BM and the lungs.

stem cells in the lung? This study and previous work document distinct intravascular and extravascular $\mathrm{MK}_{\mathrm{L}}$ populations (Figure 1) (4). Indeed, the present study shows that extravascular $\mathrm{MK}_{\mathrm{L}}$ represented approximately $60 \%$ of the total $\mathrm{MK}_{\mathrm{L}}$ population. This result raises the question of whether the $\mathrm{MK}_{\mathrm{L}}$ with an immune phenotype are mainly localized in the lung parenchyma, while $\mathrm{MK}_{\mathrm{L}}$ that are lodged in blood vessels produce platelets. Furthermore, there is the possibility that there is more than one population of $\mathrm{MK}_{\mathrm{L}}$ that could originate from different sources. Indeed, the notion of MK plasticity may further complicate these questions because of the possibility for continuous exchange of MKs or MK progenitors between the BM and lungs (Figure 1).

What is the functional consequence of having immune-like MKs in the lungs? One obvious role of the $\mathrm{MK}_{\mathrm{L}}$ could be in their response to pathogens. MHC class I molecules are found on all nucleated cells and platelets and are in charge of the presentation of cytosolic proteins to $\mathrm{CD} 8^{+}$ cytotoxic T lymphocytes. Prior work has shown that platelets (8) and MKs derived from BM (9) can present peptides through MHC class I molecules, thereby promoting $\mathrm{CD}^{+}$lymphocyte proliferation. In contrast, MHC-II molecules are found on professional antigen-presenting cells, such as dendritic cells, macrophages, and B lymphocytes, and are in charge of presentation of extracellular antigens that have been internalized and hydrolyzed in phagolysosomes. Another important finding by Pariser et al. is the identification of MHC-II on $\mathrm{MK}_{\mathrm{L}}$. The authors demonstrated that $\mathrm{MK}_{\mathrm{L}}$ were more potent than BM MKs in phagocytosis of E. coli. Moreover, using elegant in vitro and in vivo approaches, they showed that $\mathrm{MK}_{\mathrm{L}}$ presented antigens through MHC-II to specifically promote $\mathrm{CD} 4^{+} \mathrm{T}$ lymphocyte activation (6). The biological implications of $\mathrm{MK}_{\mathrm{L}}$ immune functions are interesting and far reaching, as $\mathrm{MK}_{\mathrm{L}}$ may not only have a complementary role to other lung immune cells, but may also generate a distinct pool of platelets with immune functions that have been previously overlooked.

\section{Questions of origin}

One of the looming questions that remains is whether $\mathrm{MK}_{\mathrm{L}}$ are immune cells that express MK markers or are MKs masquerading as immune cells. At its core, such cell origin questions can become almost philosophical. What makes an MK an MK? Its gene expression profile? The ability to make platelets? The data in Pariser et al. reveal common cell markers and functions between $\mathrm{MK}_{\mathrm{L}}$ and BM MKs, including the ability to produce platelets (6). As such, the data seem to point to $\mathrm{MK}_{\mathrm{L}}$ having an $\mathrm{MK}$ origin, with the lung environment modifying the cells. However, this identity question can only be definitively answered by determining if the cells of origin and subsequent differentiation trajectories of these two (or more) MK populations are the same, and if the trajecto- ries differ, where might the lineages diverge. Future studies tracing the lineage of both $\mathrm{BM}$ and $\mathrm{MK}_{\mathrm{L}}$ will be essential in establishing that location determines MK function.

Address correspondence to: Kellie R. Machlus, Karp Family Research Building, 1 Blackfan Circle, Room 11.214, Boston, Massachusetts 02115. Phone: 617.919.2389; Email: Kellie.Machlus@ childrens.harvard.edu.

1. Machlus KR, et al. The incredible journey: From megakaryocyte development to platelet formation. J Cell Biol. 2013;201(6):785-796.

2. Noetzli LJ, et al. New insights into the differentiation of megakaryocytes from hematopoietic progenitors. Arterioscler Thromb Vasc Biol. 2019;39(7):1288-1300.

3. Davis E, et al. Histologic studies of splenic megakaryocytes after bone marrow ablation with strontium 90. JLab Clin Med.1992;120(5):767-777.

4. Lefrancais E, et al. The lung is a site of platelet biogenesis and a reservoir for haematopoietic progenitors. Nature. 2017;544(7648):105-109.

5. Potts KS, et al. Membrane budding is a major mechanism of in vivo platelet biogenesis. J Exp Med. 2020;217(9):e20191206.

6. Pariser DN, et al. Lung megakaryocytes are immune modulatory cells. J Clin Invest. 2020; 131(1):e137377.

7. Fuentes R, et al. Infusion of mature megakaryocytes into mice yields functional platelets. J Clin Invest. 2010;120(11):3917-3922.

8. Chapman LM, et al. Platelets present antigen in the context of MHC class I. J Immunol. 2012;189(2):916-923.

9. Zufferey A, et al. Mature murine megakaryocytes present antigen-MHC class I molecules to $\mathrm{T}$ cells and transfer them to platelets. Blood $A d v$. 2017;1(20):1773-1785. 